\title{
The Selective Sphingosine 1-Phosphate Receptor Modulator Etrasimod Regulates Lymphocyte Trafficking and Alleviates Experimental Colitis
}

\author{
Hussien Al-Shamma, Karin Lehmann-Bruinsma, Chris Carroll, Michelle Solomon, \\ $\mathrm{H}$. Kiyomi Komori, Laurent Peyrin-Biroulet, and John Adams
}

Beacon Discovery Inc., San Diego, California (H.A.-S., K.L.-B., C.C.); Crown Bioscience, Inc., San Diego, California (M.S.); Arena Pharmaceuticals, Inc., San Diego, California (H.K.K., J.A.); and Institut National de la Santé et de la Recherche Médicale U954 and Department of Gastroenterology, Nancy University Hospital, Lorraine University, Nancy, France (L.P.-B.)

Received October 9, 2018; accepted March 5, 2019

\section{ABSTRACT}

Lymphocyte trafficking out of secondary lymphoid organs is regulated by concentration gradient-dependent interactions between the membrane-derived lysophospholipid signaling molecule sphingosine 1-phosphate (S1P) and the G-protein-coupled

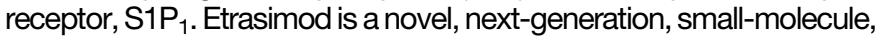
oral S1P receptor modulator in clinical development for the treatment of immune-mediated inflammatory disorders, including ulcerative colitis. In preclinical pharmacology studies, etrasimod was a full agonist of recombinant human (6.1 nM EC $\mathrm{EO}_{50}$ ), mouse (3.65 nM EC 50 ), dog (4.19 $\left.\mathrm{nM} \mathrm{EC}_{50}\right)$, and monkey (8.7 nM $\left.\mathrm{EC}_{50}\right) \mathrm{S}_{1 \mathrm{P}}$ receptors, and a partial agonist of human $\mathrm{S}^{\mathrm{P}} \mathrm{P}_{4}(147 \mathrm{nM}$ $\left.\mathrm{EC}_{50}\right)$ and $\mathrm{S}_{1} \mathrm{P}_{5}\left(24.4 \mathrm{nM} \mathrm{EC}_{50}\right)$, with relative efficacies of $63 \%$ and $73 \%$ of S1P response, respectively; whereas neither agonist nor antagonist activity was observed for human $\mathrm{S}_{1} \mathrm{P}_{2}$ or $\mathrm{S}_{1} \mathrm{P}_{3}$. A dose-dependent relationship was observed for etrasimod plasma concentration and lymphocyte count in mice, and chronic treatment with etrasimod resulted in attenuation of inflammation in a $\mathrm{CD}^{+}{ }^{+}$CD45RB ${ }^{\text {high }} \mathrm{T}$-cell transfer mouse model of colitis.

\section{Introduction}

Ulcerative colitis and Crohn's disease are debilitating inflammatory bowel diseases characterized by inappropriate and sustained immune responses (Torres et al., 2017; Ungaro et al., 2017). As a greater understanding of the underlying immune pathology of these diseases developed, treatment evolved toward a more targeted approach, with therapies aimed at proinflammatory molecules such as cytokines [e.g., tumor necrosis factor (TNF), interleukin (IL)-12/IL-23] or other biologic processes known to contribute to disease pathology, such as leukocyte trafficking (Argollo et al., 2017). Although these biologically relevant therapies have added considerable value to the treatment of the inflammatory bowel diseases, they are not equally effective in all patients, and many patients lose response to treatment over time (Billioud et al., 2011). To date, nearly all biologic therapies explored and/or approved for the treatment of inflammatory bowel disease have been developed

This work was funded by Arena Pharmaceuticals, Inc. HA-S is an employee of Beacon Discovery Inc., which is the originating laboratory for the studies; $\mathrm{KL}-\mathrm{B}$ is an employee of Beacon Discovery Inc.; CC is an employee of Beacon Discovery Inc.; MS reports; HKK is an employee of Arena Pharmaceuticals, Inc., the company that funded the studies; LP-B reports receiving honoraria from Merck, Abbvie, Janssen, Genentech, Ferring, Tillots, Vifor, Pharmacosmos, Celltrion, Takeda, Biogaran, Boerhinger-Ingelheim, Lilly, Pfizer, Index Pharmaceuticals, Amgen, Sandoz, Celgene, Biogen, Samsung Bioepis, Alma Sterna, Nestlé and Enterome; JA is an employee of Arena Pharmaceuticals, Inc. https://doi.org/10.1124/jpet.118.254268. as antibodies against potential disease targets. Paradoxically, these large molecules are themselves immunogenic, which frequently leads to altered pharmacokinetics, reduced effectiveness, and/or safety and tolerability concerns (Hindryckx et al., 2017). Moreover, the size of these molecules precludes an oral route for administration, and the costs associated with their synthesis and use are considerable. Novel, orally administered, small-molecule therapies are therefore needed (Olivera et al., 2017).

Therapies targeting molecules and pathways involved in immune cell trafficking have demonstrated efficacy in their treatment (Danese and Panés, 2014; Zundler and Neurath, 2017). A crucial component of lymphocyte circulation involves the egress of these cells out of secondary lymphoid organs and into blood and lymph (Schwab and Cyster, 2007). This process is regulated by concentration gradient-dependent interactions between the membrane-derived lysophospholipid signaling molecule sphingosine 1-phosphate (S1P) and the G-protein-coupled receptor $\mathrm{S}_{1} \mathrm{P}_{1}$ [one of five known S1P receptors $\left.\left(\mathrm{S}_{1} \mathrm{P}_{1-5}\right)\right]$ (Peyrin-Biroulet et al., 2017). Antigen-activated T cells within peripheral lymphoid organs transiently downregulate $\mathrm{S}_{1} \mathrm{P}_{1}$ and are unresponsive to $\mathrm{S} 1 \mathrm{P}$ egress signals, resulting in lymphocyte sequestration and inhibition of trafficking (Shiow et al., 2006).

Etrasimod (Fig. 1) is a synthetic next-generation S1P receptor modulator in clinical development for the treatment of immune-mediated inflammatory disorders, including ulcerative

ABBREVIATIONS: GAPDH, glyceraldehyde-3-phosphate dehydrogenase; IL, interleukin; PCR, polymerase chain reaction; PD, pharmacodynamic; $\mathrm{PK}$, pharmacokinetic; RT, room temperature; S1P, sphingosine 1-phosphate; SCID, severe combined immunodeficiency; TNF- $\alpha$, tumor necrosis factor- $\alpha$. 


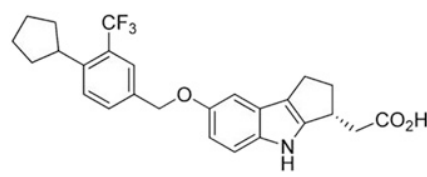

Fig. 1. Chemical structure of etrasimod: (R)-2-(7-((4-cyclo-pentyl-3(trifluoromethyl)benzyl)oxy)-1,2,3,4-tetrahydro-cyclopenta[b]indol-3yl)acetic acid).

colitis. This report describes the characterization of the preclinical in vitro pharmacology of etrasimod S1P receptor binding, as well as the pharmacodynamic and in vivo effects of etrasimod in a mouse model of experimental colitis.

\section{Materials and Methods}

Etrasimod (99.6\% purity as determined by chiral high-performance liquid chromatography) tested in the studies detailed herein was prepared in the laboratories of Arena Pharmaceuticals (San Diego, CA) as previously described (Buzard et al., 2014).

In Vitro Pharmacological Characterization of Etrasimod S1P Receptor Binding. Human recombinant $\mathrm{S} \mathrm{P}_{1-5}$ and mouse, dog, and monkey $\mathrm{S}_{1} \mathrm{P}_{1}$ receptors were stably expressed in PathHunter HEK293 (DiscoveRx, Freemont, CA) parental cell lines after confirmation of cDNA accuracy against current National Center for Biotechnology Information reference sequences. Agonist $\left(\mathrm{S}_{1} \mathrm{P}_{1-5}\right)$ and antagonist $\left(\mathrm{S}_{2} \mathrm{P}_{2}\right.$ and $\left.\mathrm{S}_{1} \mathrm{P}_{3}\right) \beta$-arrestin recruitment assays were performed according to the manufacturer (DiscoveRx) instructions. Briefly, PathHunter (DiscoveRx) HEK293 cells stably expressing recombinant receptors were seeded onto 384-well microtiter plates (2000-5000 cells/well depending on receptor) in OptiMem (Thermo Fisher Scientific, Waltham, MA) containing $0.05 \%$ fatty acid-free bovine serum albumin and incubated overnight in a humidified chamber. Plates were then equilibrated to room temperature (RT) for 1 hour followed by the addition of $5 \mu \mathrm{l}$ of at least 10 different etrasimod concentrations $(<10 \mu \mathrm{M})$ and incubation at RT for $2-3$ hours. Lysis/detection reagents (12 $\mu \mathrm{l}$ total) were then added, and the plates were sealed and incubated for an additional 2 hours at RT. Plates were read on an EnVision (Perkin Elmer, San Jose, CA) or PheraStar (BMG, Cary, NC) plate reader. All etrasimod $\mathrm{EC}_{50}$ values were determined with a minimum of 10 different concentrations in triplicate. An $\mathrm{EC}_{90}$ of 200 and $300 \mathrm{nM} \mathrm{S1P}$ was added to plates 10 minutes after the addition of etrasimod for $\mathrm{S}_{2} \mathrm{P}_{2}$ and $\mathrm{S}_{3} \mathrm{P}_{3}$ antagonist assays, respectively.

In Vivo Regulation of Lymphocyte Counts in Mice. Adult male Balb/c mice (Harlan, Indianapolis, IN) $(N=252)$ were used in this study and housed in climate-controlled conditions with a 12-hour automatic light/dark cycle with free access to certified rodent diet and water. All of the in vivo study protocols were reviewed and approved by the Arena Pharmaceuticals Institutional Animal Care and Use Committee.

Mice weighing $24.3 \pm 2.1 \mathrm{~g}$ (mean \pm S.D.) were randomly assigned to vehicle $(n=48)$, or $0.03,0.10,0.30$, or $1.0 \mathrm{mg} / \mathrm{kg}$ etrasimod dose groups ( $n=51$ per dose) and received a single oral gavage dose (in the fed state) of either vehicle $(0.5 \%$ methylcellulose) or etrasimod at a volume of $10 \mathrm{ml} / \mathrm{kg}$. Whole-blood samples were collected by cardiac puncture and treated with potassium-EDTA. Plasma was prepared by centrifugation and stored frozen $\left(-80^{\circ} \mathrm{C}\right)$ until analysis. The total lymphocyte count in whole blood was analyzed within 30 minutes of collection at $1,3,5,8,16,24$, and 32 hours postdose using an automated laser hematology analyzer (CELL-DYN 3700; Abbott Laboratories, Abbott Park, IL) designed for in vitro diagnostic use in clinical laboratories.

Plasma etrasimod concentrations at $0.25,0.5,1,2,3,5,8,16,24$, and 32 hours postdose were analyzed using a selective liquid chromatography-mass spectrometry method. Plasma proteins were extracted with acetonitrile in the presence of an internal standard followed by centrifugation at $3700 \mathrm{rpm}$ for 20 minutes. The supernatant from the processed plasma samples was injected into a high-performance liquid chromatography system equipped with either an Applied Biosystems API 4000 or API 5000 mass spectrometer (Thermo Fisher Scientific). Peak areas for the transitions of mass/charge ratio $(\mathrm{m} / \mathrm{z}) 458.2 \rightarrow 159.3$ product ion of etrasimod were measured against the $\mathrm{m} / z 388.9 \rightarrow 278.1$ product ion of the internal standard, in positive-ion multiple reaction-monitoring mode.

Experimental Colitis Model. Female, 9-week-old CB17/IcrPrkdcscid/IcrCr mice ( $N=45$; strain 561; Charles River Laboratories, San Diego, CA) were housed in climate- and lighting-controlled conditions (reverse light/dark cycle, lights off 11:30-19:30) and allowed 1 week of habituation to this environment prior to study initiation. Animals had free access to sterilized rodent chow (2018 Teklad; Harlan) and water.

Spleens were dissected from BALB/c female donor mice (Charles River Laboratories) and placed in a staining buffer $\left[\mathrm{PBS} \mathrm{Ca} / \mathrm{Mg}^{2+}\right.$ free; $1 \mathrm{mM}$ EDTA, $25 \mathrm{mM}$ HEPES ( $\mathrm{pH} 7$ ), 4\% FBS (heat inactivated); $10 \mathrm{U} / \mathrm{ml}$ DNase II]. Single-cell suspension was generated by mashing spleens through 70- $\mu \mathrm{m}$ nylon mesh strainers (Falcon; Thermo Fisher Scientific). Cells were pelleted and suspended 1:3 in Life Technologies ACK (ammonium-chloride-potassium) lysis buffer (Thermo Fisher Scientific) for 3-5 minutes at RT followed by the addition of cold staining buffer. Cells were pelleted and suspended in MACS buffer (PBS, 0.5\% BSA, 2 mM EDTA, pH 7.2; Miltenyi Biotec, San Diego, CA). $\mathrm{CD}^{+}{ }^{+} \mathrm{T}$ cells were enriched by negative selection using a CD4 ${ }^{+} \mathrm{T}$-cell isolation kit (Miltenyi Biotec) and LS magnetic columns (Miltenyi Biotech). Briefly, $10^{7}$ cells were suspended in $40 \mu$ l of ice-cold MACS buffer to which $10 \mu$ l of biotin-antibody cocktail was added, mixed, and incubated on ice for 5 minutes. An additional $30 \mu \mathrm{l}$ of ice-cold MACS buffer was added, as well as $20 \mu \mathrm{l}$ of anti-biotin microbeads, followed by incubation on ice for 10 minutes. Cells, antibody, and bead suspensions were then added to LS columns (Miltenyi Biotech) previously rinsed with $3 \mathrm{ml}$ of MACs buffer. The flow through was collected, and cells were centrifuged and resuspended in $100 \mu \mathrm{l}$ of basic sorting buffer [PBS $\mathrm{Ca} / \mathrm{Mg}^{2+}$ free; $1 \mathrm{mM}$ EDTA, $25 \mathrm{mM}$ HEPES (pH 7), $4 \%$ heat-inactivated FBS; $10 \mathrm{U} / \mathrm{ml}$ DNase II; $0.2 \mu \mathrm{M}$ filter sterilized] per $10^{6}$ cells. Monoclonal antibodies (CD45RB fluorescein isothiocyanate; CD4 APC; CD25 PE; all from BD Biosciences) were added and incubated on ice for 30 minutes in the dark.

Cells were washed with ice-cold staining buffer and stained with $1 \mu \mathrm{M} \mathrm{4}$ ',6-diamidino-2-phenylindole. The 4',6-diamidino-2phenylindole-negative, $\mathrm{CD} 4^{+}$, and $\mathrm{CD} 45 \mathrm{RB}^{\text {high }}$ cells were sorted on an Astrios (100 $\mu \mathrm{M}, 25$ psi; Beckman Coulter Life Sciences, Indianapolis, IN) into $50 \%$ FBS, centrifuged at $3000 \mathrm{rpm}$ for 15 minutes and suspended in ice-cold PBS $\left(\mathrm{Ca}, \mathrm{Mg}^{2+}\right.$ free $)$ at $1 \times 10^{6}$ cells $/ \mathrm{ml}$. A total of $5 \times 10^{5}$ cells were intraperitoneally injected into female severe combined immunodeficiency (SCID) mice. SCID mice injected

\section{TABLE 1}

Etrasimod mean effective concentrations and efficacy on recombinant S1P receptors by $\beta$-arrestin assay

\begin{tabular}{lccc}
\hline Receptor $^{a}$ & Mean $^{b} \mathrm{EC}_{50}$ & Mean Efficacy & Replicates \\
\hline & $\mathrm{nM}$ & \% of Activity & $n$ \\
$\mathrm{~S}_{1} \mathrm{P}_{1}$ & & & \\
Human & & & \\
Mouse & $6.1(3.36,11.1)$ & 110 & 6 \\
Dog & $4.65(2.06,6.44)$ & 82 & 6 \\
Monkey & $8.70(6.59,7.02)$ & 101 & 6 \\
$\mathrm{~S}_{2}$ & $>10,000$ & 88 & 6 \\
$\mathrm{~S}_{2}$ & $>10,000$ & $\mathrm{ND}$ & 8 \\
$\mathrm{~S}_{3}$ & $147(114,190)$ & $\mathrm{ND}$ & 6 \\
$\mathrm{~S}_{4}$ & $24.4(17.4,34.3)$ & 73 & 9 \\
& & & \\
\hline
\end{tabular}

$\mathrm{ND}$, not determined.

${ }^{a}$ All receptors are human recombinant unless otherwise noted.

${ }^{b}$ All $95 \%$ confidence intervals are reported in parentheses.

${ }^{c}$ From Buzard et al. (2014). 
A
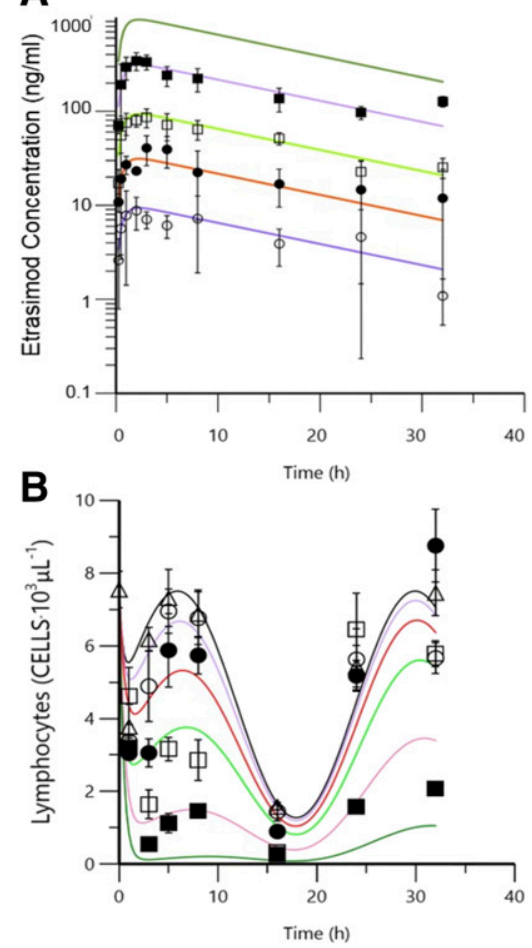

Fig. 2. Comparison of mean etrasimod plasma concentration and blood lymphocyte count vs. time profiles. Dose-dependent increases in plasma concentration plotted over time (A) are inversely related to peripheral blood lymphocyte counts over the same time period (B). Note: collected data were used to build a PK/PD model that was then used to derive the predicted exposure and associated lymphocyte counts represented by the continuous line plots. MC; methylcellulose.

with $5 \times 10^{5}$ unsorted enriched T cells served as baseline controls. SCID mice injected with colitogenic T cells were orally dosed daily beginning on the day following transfer until the day before tissue harvest (Day 32) with either vehicle (negative control; $n=11) 1(n=12)$ or $3 \mathrm{mg} / \mathrm{kg}(n=12)$ etrasimod or $1 \mathrm{mg} / \mathrm{kg}$ fingolimod (positive control; $n=12$ ) in a volume of $4 \mathrm{ml} / \mathrm{kg}$. All mice were weighed daily.

Prior to tissue harvest, blood was collected and mice were anesthetized with isoflurane and then euthanized. Colon segments were dissected, measured, and flushed with saline prior to weighing. Approximately $4 \mathrm{~cm}$ of the distal colon was fixed in formalin for 48 hours at RT, dehydrated, paraffin embedded, and sectioned (10 $\mu \mathrm{m}$ thickness), followed by staining with H\&E. The remaining colon tissues was frozen in liquid nitrogen and stored at $-80^{\circ} \mathrm{C}$ for RNA isolation.

Frozen tissue was subsequently homogenized in tubes containing lysing matrix A (MP Biomedicals, Santa Ana, CA) with $1 \mathrm{ml}$ of TRIzol (Thermo Fisher Scientific) using an MP FastPrep homogenizing machine (MP Biomedicals). Ultrapure phenol:chloroform:isoamyl alcohol (200 ml; Thermo Fisher Scientific) was immediately added. Samples were incubated at RT for 10 minutes, followed by centrifugation at $15,000 \mathrm{rpm}$ for 15 minutes at $4^{\circ} \mathrm{C}$. The upper clear phase was removed and placed in a new tube containing $500 \mu \mathrm{l}$ of isopropanol, followed by incubation for 10 minutes at RT and centrifugation at $15,000 \mathrm{rpm}$ for 15 minutes. The RNA pellet was washed once with $70 \%$ ethanol, and centrifuged for 15 minutes at $15,000 \mathrm{rpm}$ before reconstitution in RNase/DNase-free water. Quantitation of RNA was performed with NanoDrop Lite (Thermo Fisher Scientific) prior to treatment with DNase (Thermo Fisher Scientific) and reverse transcription using iScript cDNA Synthesis Kit (Bio-Rad, Hercules CA). Quantitative polymerase chain reaction (PCR) was performed using QuantStudio 6 (Thermo Fisher Scientific) for CD4 (Mm00442754_m1), CD3 $\gamma$ (Mm00438095_m1), CD11b (Mm00434455_m1), IL-17A (Mm00439618_m1), IL-13 (Mm00434204_m1), interferon- $\gamma$ (Mm01168134_m1), TNF- $\alpha$ (Mm00443258_m1), IL-1 $\beta$ (Mm00434228_m1), IL-6(Mm00446190_m1), and IL-10 (Mm00439616_m1; all Thermo Fisher Scientific); and Ly6G (Beacon Discovery, San Diego, CA).

For the assessment of histologic disease severity, blinded histopathological assessment was performed using a modification of previously described methods (Ostanin et al., 2006; Pavlick et al., 2006). Briefly, the following scoring criteria were assessed in a blinded fashion by a single pathologist: 1) crypt score based on abnormal crypt architecture including distortion, branching, atrophy, and crypt loss (range, 0-3); 2) goblet cell loss (range, 0-2); and 3) mucosal erosion and ulceration (range, 0-1), and 4) immune cell infiltration (range, 0-2). A total histopathological score is calculated by combining the scores for each of the four parameters for a maximum score of 8 .

Data Analysis. For receptor assays, efficacies were calculated as a percentage of the $\mathrm{S} 1 \mathrm{P}$ receptor dose-response curve height, which was defined as $100 \%$ in each experiment. Mean EC $_{50}$ values were calculated from mean $\mathrm{pEC}_{50}$.

Composite sampling was used to determine the blood lymphocyte and plasma concentration versus time profiles. The relationship between

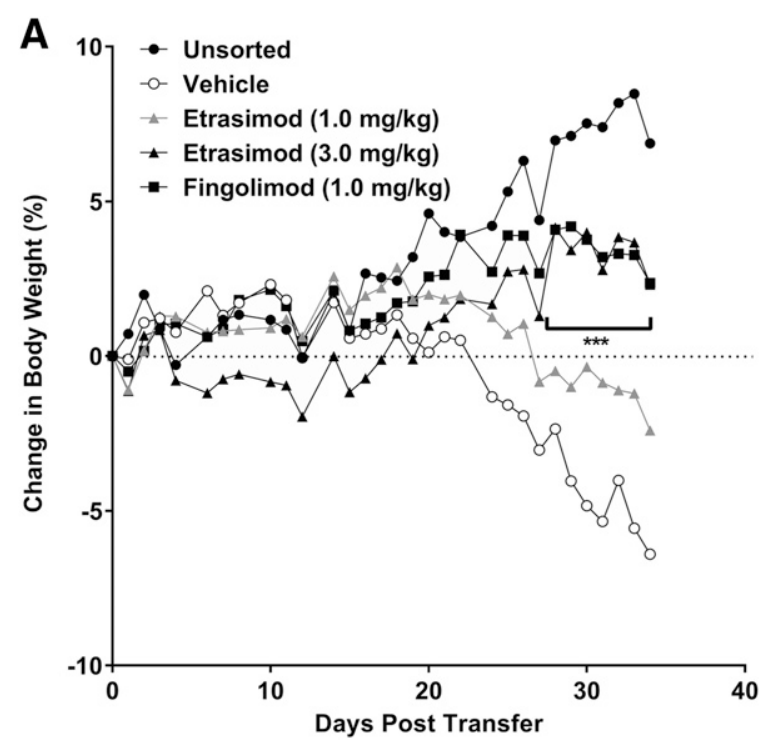

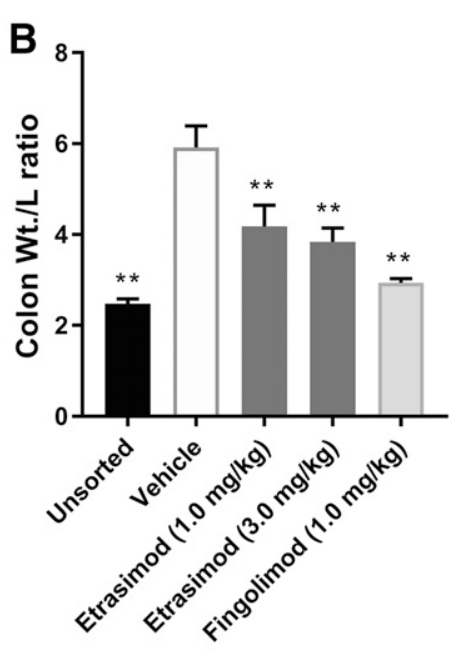

Fig. 3. Treatment with etrasimod or fingolimod inhibits weight loss associated with disease onset in SCID mice receiving $\mathrm{CD}^{+}$ colitogenic T cells. Etrasimod attenuates weight loss (A) and reduces colon weightto-length ratio (B) dose-dependently compared with vehicle-treated controls (vs. vehicle: $* * P \leq 0.01 ; * * * P \leq 0.001$ Dunnett's test). 
etrasimod plasma concentration and lymphocyte count was determined using an indirect pharmacokinetic (PK)/pharmacodynamic (PD) model. Initial observations indicated that the blood lymphocyte count was associated with a circadian rhythm. To discern between the lymphocyte baseline diurnal effect and the effect of etrasimod on lymphocyte egress, a circadian equation was built into the model. The relationship between drug concentration and pharmacological effect were expressed by model estimates $\mathrm{IC}_{50}$ and $I_{\max }$. These values were determined by simultaneously modeling the blood lymphocyte counts over time for all doses (including vehicle control) while holding the PK parameters constant. This PK/PD model was also used to calculate plasma concentrations and associated lymphocyte counts for a $3 \mathrm{mg} / \mathrm{kg}$ dose to provide estimates for the dose used in the adoptive transfer study.

Body weight was expressed as a percentage of initial body weight at the day of T-cell transfer and subjected to ANOVA followed by Dunnett's multiple-comparison test with all groups compared with vehicle control. For quantitative PCR, mRNA level was first normalized to glyceraldehyde-3-phosphate dehydrogenase (GAPDH) level within each sample to obtain a ratio, all values were then normalized to the average of values from naive SCID mice, and expressed as a fold value over control (naive). One-way ANOVA followed by Dunnett's test was used to compare multiple groups. All analyses were done with GraphPad PRISM 7 (2016).

\section{Results}

S1P Receptor Binding. Etrasimod has previously been shown to be a full agonist of human $\mathrm{S}_{1} \mathrm{P}_{1}$ and a partial agonist of $\mathrm{S} \mathrm{P}_{4}$ and $\mathrm{S}_{1} \mathrm{P}_{5}$ in a $\beta$-arrestin assay; and a full agonist of human, mouse, rat, dog and monkey $\mathrm{S} \mathrm{P}_{1}$ demonstrated in a cAMP assay (Buzard et al., 2014). Here, we further demonstrate that etrasimod is a full agonist of human, dog, mouse, and monkey recombinant $\mathrm{S}_{1} \mathrm{P}_{1}$ receptors in $\beta$-arrestin recruitment assays (Table 1). Additionally, neither agonist nor antagonist activity was observed for etrasimod on either the human recombinant $\mathrm{S}_{1} \mathrm{P}_{2}$ or $\mathrm{S}_{1} \mathrm{P}_{3}$ receptors. The mean $\mathrm{EC}_{50}$ values of etrasimod for $\mathrm{S}_{1} \mathrm{P}_{1}$ were similar among the species tested and ranged from 3.65 to $8.70 \mathrm{nM}$. Selectivity for human $\mathrm{S}_{1} \mathrm{P}_{1}$ was 24 -fold and 4 -fold compared with $\mathrm{S}_{1} \mathrm{P}_{4}$ and $\mathrm{S}_{1} \mathrm{P}_{5}$, respectively, and $\geq 1000$-fold compared with $\mathrm{S}_{1} \mathrm{P}_{2}$ and $\mathrm{S}_{1} \mathrm{P}_{3}$.

Effect of Etrasimod on Lymphocyte Counts. Etrasimod was rapidly absorbed after oral administration, with quantifiable plasma concentrations detected at the first time point measured ( 0.25 hour), and subsequent dose-proportional increases were observed for all doses tested.

Etrasimod produced dose-dependent blood lymphopenia in mice, and plasma etrasimod concentrations and lymphocyte counts were inversely related (Fig. 2 ). The calculated $\mathrm{IC}_{50}$ value was $46.3 \mathrm{ng} / \mathrm{ml}$ (planar 95\% confidence interval, -13.7 to 106), which is equivalent to a median effective dose of approximately $0.2 \mathrm{mg} / \mathrm{kg}$. The $I_{\max }$ of lymphocyte egress based on model estimates was approximately 1.03 (planar $95 \%$ confidence interval, $0.686-1.36$ ) or $100 \%$. Additional model estimates suggest a further blunting of lymphocyte egress after a $3 \mathrm{mg} / \mathrm{kg}$ dose. Also of note is a clear dose-dependent blunting of the circadian rhythm associated with lymphocyte trafficking.

Attenuation of Disease in Mouse Colitis Model. Onset of progressive colitis-like symptoms (loose, mucinous stool and loss of body weight) began between 2 and 3 weeks after adoptive transfer of $\mathrm{CD} 4^{+} \mathrm{CD} 45 \mathrm{RB}^{\text {high }} \mathrm{T}$ cells. Mice that received colitogenic $T$ cells showed significant weight loss over the course of the study compared with controls treated with unsorted T cells. Etrasimod treatment dose-dependently attenuated this loss, with a statistically significant effect observed at $3 \mathrm{mg} / \mathrm{kg}$. Similarly, fingolimod treatment significantly inhibited weight loss compared with vehicle-treated controls (Fig. 3A).

Compared with naive mice and mice who received unsorted $\mathrm{CD} 4 \mathrm{~T}$ cells, $\mathrm{CD} 4{ }^{+} \mathrm{CD} 45 \mathrm{RB}^{\text {high }}$ SCID mice treated with vehicle had an increase in colon weight:length ratio. Treatment with 1 and $3 \mathrm{mg} / \mathrm{kg}$ etrasimod and $1 \mathrm{mg} / \mathrm{kg}$ fingolimod significantly inhibited the increase in colon weight:length ratio in $\mathrm{CD}^{+}{ }^{+} \mathrm{CD} 45 \mathrm{RB}^{\text {high }}$ SCID mice (Fig. 3B).

Consistent with the increased colon weight:length ratio, histopathologic examination suggested that the colonic mucosae of $\mathrm{CD} 4{ }^{+} \mathrm{CD} 45 \mathrm{RB}^{\text {high }}$ SCID mice were thicker than those of naive SCID mice and mice who had received unsorted $\mathrm{T}$ cells. Treatment with etrasimod $(3 \mathrm{mg} / \mathrm{kg})$ or fingolimod $(1 \mathrm{mg} / \mathrm{kg})$ resulted in significant reductions in mucosal thickness (Fig. 4A), and lower histopathology scores (Fig. 4B). These differences are readily observable in photomicrographs of colon cross sections from the respective groups (Fig. 4C) wherein etrasimod and fingolimod reduce the number of inflammatory cell
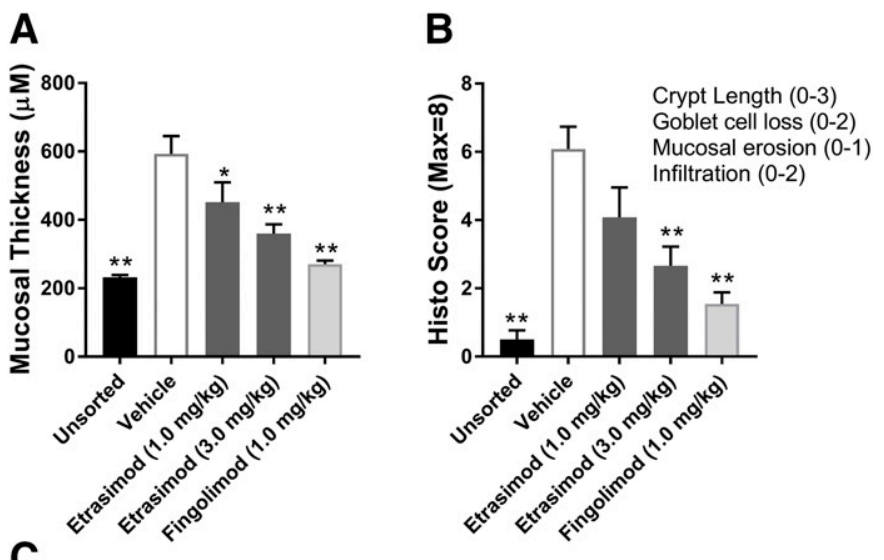

C

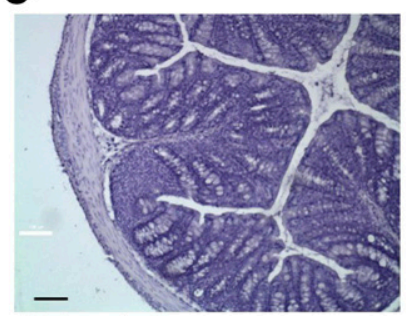

Untreated

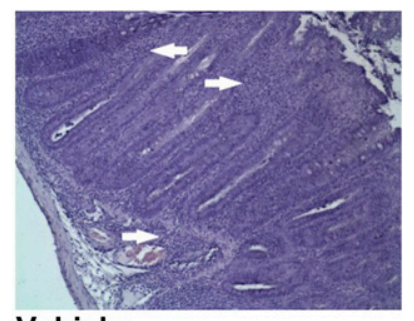

Vehicle

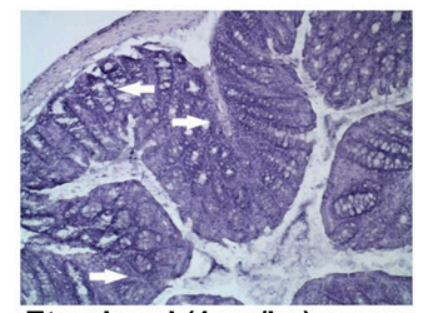

Etrasimod (1mg/kg)

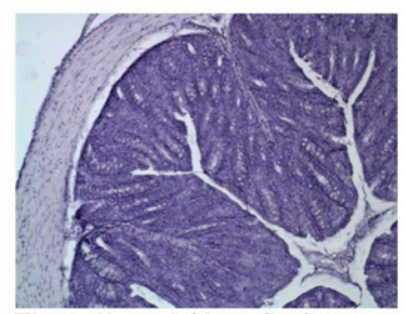

Fingolimod (1 $\mathrm{mg} / \mathrm{kg})$

Fig. 4. Etrasimod inhibits the increase in histologic (Histo) markers of disease. (A) Etrasimod inhibits increases in mucosal thickening. (B) Etrasimod reduces the increase in disease index scores. (C) Photomicrographs of H\&E-stained colon cross sections suggest that etrasimod decreases inflammatory cell infiltration (arrows) in the colons of mice transferred with colitogenic $\mathrm{T}$ cells (vs. vehicle: $* P \leq 0.05 ; * * P \leq 0.01$ Dunnett's test). Max, maximum. 
infiltrates compared with vehicle-treated mice transferred with colitogenic $\mathrm{T}$ cells.

Reduction in Inflammatory Responses in Mouse Colitis Model. Increased expression of immune cell markers and cytokines as estimated by quantitative PCR was observed in the colonic tissue isolated from $\mathrm{CD} 4{ }^{+} \mathrm{CD} 45 \mathrm{RB}^{\text {high }}$ SCID mice and mice that had received unsorted $\mathrm{T}$ cells compared with naive mice, with comparably higher levels of CD4 (T cells, natural killer cells, and dendritic cells), CD3 $\gamma$ (T lymphocytes) and CD11b (monocytes, natural killer cells, and dendritic cells), and unchanged levels of Ly6G (neutrophils, and granulocytes) observed in $\mathrm{CD}^{+}{ }^{+} \mathrm{CD} 45 \mathrm{RB}^{\text {high }}$ SCID mice (Fig. 5). Treatment with etrasimod ( $3 \mathrm{mg} / \mathrm{kg}$ ) or fingolimod ( $1 \mathrm{mg} / \mathrm{kg})$ significantly reduced the expression of the T-cell and monocyte markers, suggesting that both agents decreased infiltration and expansion of these cell populations in the colons of CD $4^{+} \mathrm{CD} 45 \mathrm{RB}^{\text {high }}$ SCID mice.

In support of these data, T-cell and/or monocyte-derived proinflammatory cytokines TNF- $\alpha$, IL-1 $\beta$, IL-6, and IL-17A were also significantly lower in $\mathrm{CD}^{+}{ }^{+} \mathrm{CD} 45 \mathrm{RB}^{\text {high }}$ SCID mice treated with etrasimod $(3 \mathrm{mg} / \mathrm{kg})$ and fingolimod $(1 \mathrm{mg} / \mathrm{kg})$ compared with mice treated with vehicle (Fig. 6). Etrasimod induced dose-dependent increases in the anti-inflammatory cytokine IL-10. Expression levels of this cytokine were comparable between animals treated with the high dose of etrasimod and with fingolimod, and both were significantly higher than those in the vehicle-treated control group $(P<0.05$ Dunnett's test $)$
(Fig. 6). Last, no clear differences between the treatment groups were observed in both IL-13 and interferon- $\gamma$ expression (data not shown).

\section{Discussion}

In this report, we further characterize the preclinical in vitro pharmacology of etrasimod S1P receptor binding, as well as the pharmacodynamic and in vivo effects of etrasimod in a mouse model of experimental colitis. The data demonstrate that etrasimod is a full agonist of $\mathrm{S}_{1} \mathrm{P}_{1}$ across multiple species with greater than 1000 -fold selectivity versus human $\mathrm{S}_{2} \mathrm{P}_{2}$ and $\mathrm{S}_{3} \mathrm{P}_{3}$, with neither agonist nor antagonist activity observed for these latter receptors. Etrasimod was rapidly absorbed after oral administration and produced dosedependent reductions in lymphocyte counts in mice, which were inversely related to etrasimod plasma concentrations. Consistent with previous reports, there was a clear circadian pattern in lymphocyte egress as measured with lymphocyte counts, which also showed a dose-dependent response to increasing concentrations of circulating etrasimod (Druzd et al., 2017). These circadian fluctuations were almost completely blunted at higher doses of etrasimod. Importantly, oral administration of etrasimod at a dose of $3 \mathrm{mg} / \mathrm{kg}$ was efficacious in a mouse model of colitis, demonstrating attenuation of colitis-like symptoms and reductions in mucosal thickness and histologic disease activity scores. These outcomes were likely a
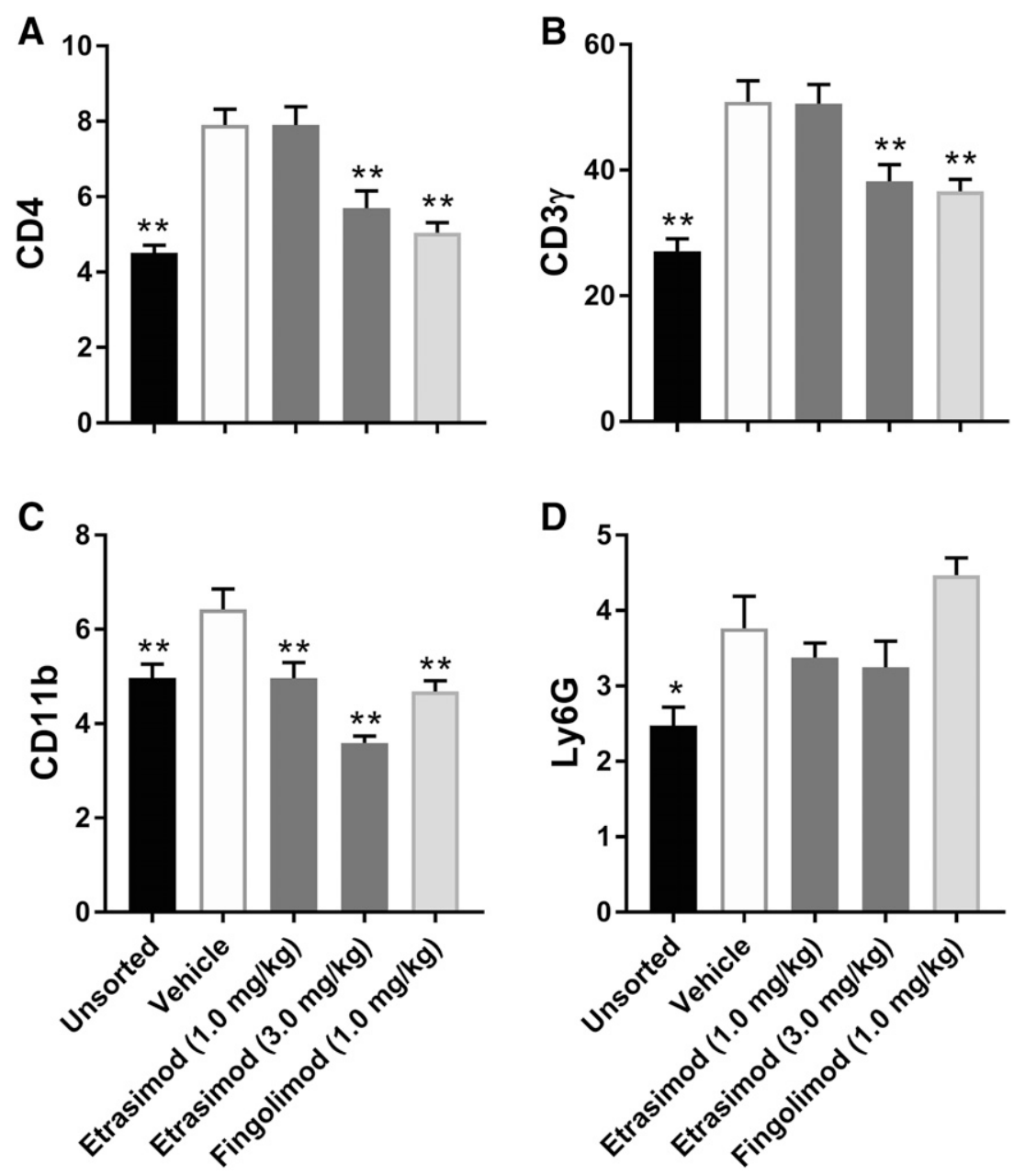

Fig. 5. Etrasimod reduces infiltration and expansion of T cell and macrophage populations. QPCR of colon homogenates shows statistically significant decreases in (A) CD4, (B) CD3 $\gamma$ and (C) CD11b, but not (D) Ly6G in mice treated with etrasimod. Data shown as relative expression to GAPDH. (vs. vehicle $* P<0.05$; $* * P<0.01$. Dunnet's). 

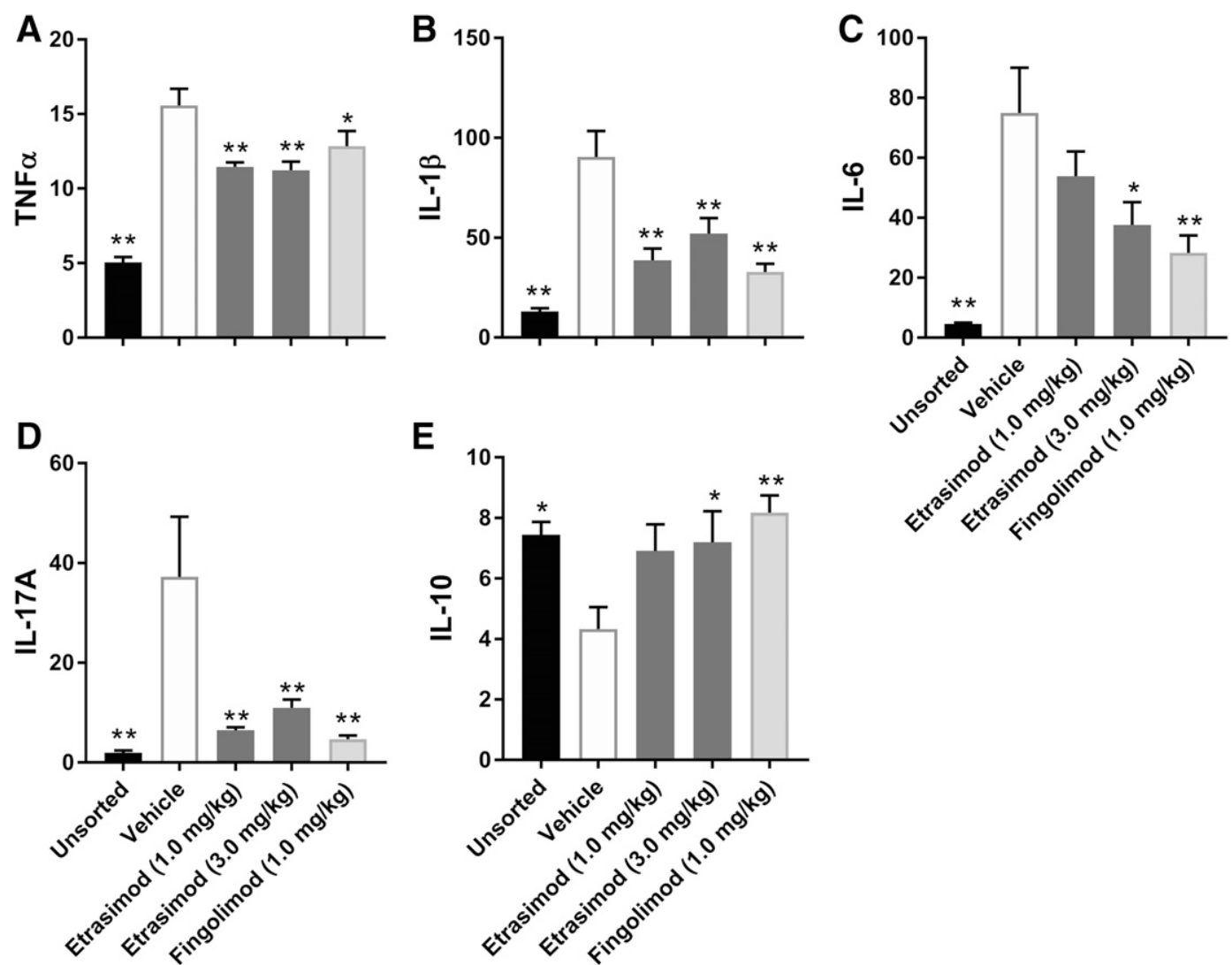

result of the inhibitory effects of etrasimod observed on the expression of proinflammatory cytokines (TNF- $\alpha$, IL- $1 \beta$, IL-6, IL-17A) and increased anti-inflammatory IL-10 expression. It is important to note that etrasimod treatment in the mouse colitis model did not affect the expression of the neutrophil cell surface marker, Ly6G, suggesting that innate immune surveillance by these cells is likely protected with etrasimod treatment.

Proof-of-concept support for $\mathrm{S} 1 \mathrm{P}$ receptor targeting in chronic inflammatory diseases was first demonstrated in clinical trials of fingolimod, a nonselective S1P modulator approved for the treatment of relapsing multiple sclerosis (Calabresi et al., 2014). Adverse effects of fingolimod, such as macular edema, dysregulated pulmonary function, and hypertension, however, are presumed to stem from the nonspecific $\mathrm{S}_{1} \mathrm{P}_{1-5}$ activity (Forrest et al., 2004; Camm et al., 2014). $\mathrm{S}_{2} \mathrm{P}_{2}$ and $\mathrm{S} \mathrm{P}_{3}$ signaling are implicated in a myriad of biologic activities (Sanna et al., 2004; Blaho and Hla, 2014). Although a complete understanding of the specific biologic functions mediated by the individual receptors is lacking, in general, $\mathrm{S}_{1} \mathrm{P}_{1}, \mathrm{~S}_{1} \mathrm{P}_{4}$, and $\mathrm{S}_{1} \mathrm{P}_{5}$ appear to be fundamentally involved in the regulation of the immune system (Blaho and Hla, 2014), whereas $\mathrm{S}_{2} \mathrm{P}_{2}$ and $\mathrm{S} \mathrm{P}_{3}$ have been implicated in processes such as vasoconstriction and fibrosis, mechanisms that may play a role in the renal ischemia-reperfusion injury potentially observed with $\mathrm{S}_{2} \mathrm{P}_{2}(\mathrm{Park}$ et al., 2012) and the hypertension observed with fingolimod (Fryer et al., 2012). More selective S1P receptor modulators (ozanimod, siponimod, ponesimod) in clinical development have demonstrated improved clinical safety profiles compared with fingolimod (Piali et al., 2011; Gergely et al., 2012; Tran et al., 2018). In this regard, the receptor-binding profile of etrasimod should theoretically avoid some of the adverse effects associated with modulation of these receptors, while simultaneously decreasing intestinal inflammation.

The selectivity of etrasimod for $\mathrm{S}_{1} \mathrm{P}_{1}$ has important and specific disease-related implications for the treatment of inflammatory bowel disease. In a study designed to investigate the role of the S1P pathway on inflammatory bowel disease pathogenesis, Karuppuchamy et al. (2017) found $\mathrm{S}_{1} \mathrm{P}_{1}$ expressed on naive, central memory cells and subsets of gut-homing effector T cells, activated dendritic cells, and endothelial cells. Furthermore, chronic inflammatory signals were shown to upregulate $\mathrm{S}_{1} \mathrm{P}_{1}$ on both $\mathrm{T}$ cells and the endothelium, and a similar pattern of dysregulation was observed for enzymes that control tissue S1P levels in inflamed mouse and human intestines (i.e., induction of S1P synthesis and suppression of degradation). Karuppuchamy et al. (2017) hypothesized that $\mathrm{S} \mathrm{P}_{1}$ inhibitors could have multiple relevant anti-inflammatory mechanisms of action in the treatment of inflammatory bowel disease apart from their lymphopenic effects, including potential effects on dendritic cell migration and vascular barrier function (Karuppuchamy et al., 2017). Initial clinical results for selective S1P receptor modulators in the treatment of inflammatory bowel disease have been encouraging. The $\mathrm{S} 1 \mathrm{P}$ receptor modulator ozanimod, which targets both $\mathrm{S}_{1} \mathrm{P}_{1}$ and $\mathrm{S}_{1} \mathrm{P}_{5}$, has demonstrated efficacy 
and tolerability in phase 2 clinical trials of ulcerative colitis (Sandborn et al., 2016) and in phase 3 clinical trials of multiple sclerosis (Cohen et al., 2017; Comi et al., 2017). However, longerterm data and larger trials are required to fully characterize the efficacy and safety profile of this agent in patients with inflammatory bowel disease.

In conclusion, etrasimod is a second-generation, oral, synthetic small-molecule $\mathrm{S} 1 \mathrm{P}$ receptor modulator that may address some of the limitations associated with currently approved monoclonal antibodies (i.e., route of administration, immunogenicity, prolonged half-lives, and manufacturing costs). Etrasimod receptor selectivity $\left(\mathrm{S}_{1} \mathrm{P}_{1,4,5}\right)$ may avoid off-target effects related to broader receptor binding, such as those observed with fingolimod, and provide relevant mechanistic effects specifically related to the role of $\mathrm{S}_{1} \mathrm{P}_{1}$ in the pathology of inflammatory bowel disease. Furthermore, an oral route of administration may provide additional convenience and options to patients and their treating gastroenterologists. Future clinical trials in humans will provide more efficacy and safety data on etrasimod and its potential placement in the treatment armamentarium for ulcerative colitis.

\section{Authorship Contributions}

Participated in research design: Al-Shamma, Lehmann-Bruinsma, Carroll, Solomon, and Adams.

Conducted experiments: Al-Shamma, Lehmann-Bruinsma, Carroll, and Solomon.

Performed data analysis: Al-Shamma, Lehmann-Bruinsma, Carroll, and Solomon.

Wrote or contributed to the writing of the manuscript: Al-Shamma, Komori, Peyrin-Biroulet, and Adams.

\section{References}

Argollo M, Fiorino G, Hindryckx P, Peyrin-Biroulet L, and Danese S (2017) Novel therapeutic targets for inflammatory bowel disease. J Autoimmun 85:103-116.

Billioud V, Sandborn WJ, and Peyrin-Biroulet L (2011) Loss of response and need for adalimumab dose intensification in Crohn's disease: a systematic review. Am $J$ Gastroenterol 106:674-684.

Blaho VA and Hla T (2014) An update on the biology of sphingosine 1-phosphate receptors. J Lipid Res 55:1596-1608.

Buzard DJ, Kim SH, Lopez L, Kawasaki A, Zhu X, Moody J, Thoresen L, Calderon I, Ullman B, Han S, et al. (2014) Discovery of APD334: design of a clinical stage functional antagonist of the sphingosine-1-phosphate-1 receptor. ACS Med Chem Lett 5:1313-1317.

Calabresi PA, Radue EW, Goodin D, Jeffery D, Rammohan KW, Reder AT, Vollmer T, Agius MA, Kappos L, Stites T, et al. (2014) Safety and efficacy of fingolimod in patients with relapsing-remitting multiple sclerosis (FREEDOMS II): a doubleblind, randomised, placebo-controlled, phase 3 trial. Lancet Neurol 13:545-556.

Camm J, Hla T, Bakshi R, and Brinkmann V (2014) Cardiac and vascular effects of fingolimod: mechanistic basis and clinical implications. Am Heart $J$ 168:632-644.

Cohen J, Comi G, Selmaj K, Bar-Or A, Arnold D, Douglas Steinman L, Hartung H-P, Montalbán X, Havrdová E, Cree B, et al. (2018) Clinical and magnetic resonance imaging results from RADIANCE part B, a multicenter, randomized, double-blind, phase 3 trial of ozanimod versus intramuscular interferon $\beta-1 \mathrm{a}$ in relapsing multiple sclerosis (RMS). Neurology 90 (15 Supplement). (https://n.neurology.org/ content/90/15_Supplement/P3.410).

Comi G, Arnold D, Cree B, Kappos L, Selmaj K, Bar-Or A, Steinman L, Hartung H-P Montalbán X, Havrdová E, et al. (2018) Ozanimod demonstrates efficacy and safety in a Phase 3 trial of relapsing multiple sclerosis (SUNBEAM). Neurology 90 (15 Supplement). (https://n.neurology.org/content/90/15_Supplement/P3.396).

Danese S and Panés J (2014) Development of drugs to target interactions between leukocytes and endothelial cells and treatment algorithms for inflammatory bowel diseases. Gastroenterology 147:981-989.

Druzd D, Matveeva O, Ince L, Harrison U, He W, Schmal C, Herzel H, Tsang AH Kawakami N, Leliavski A, et al. (2017) Lymphocyte circadian clocks control lymph node trafficking and adaptive immune responses. Immunity 46:120-132.

Forrest M, Sun SY, Hajdu R, Bergstrom J, Card D, Doherty G, Hale J, Keohane C, Meyers C, Milligan J, et al. (2004) Immune cell regulation and cardiovascular effects of sphingosine 1-phosphate receptor agonists in rodents are mediated via distinct receptor subtypes. J Pharmacol Exp Ther 309:758-768.

Fryer RM, Muthukumarana A, Harrison PC, Nodop Mazurek S, Chen RR, Harrington KE, Dinallo RM, Horan JC, Patnaude L, Modis LK, et al. (2012) The clinically-tested S1P receptor agonists, FTY720 and BAF312, demonstrate subtypespecific bradycardia $\left(\mathrm{S}_{\mathrm{P}} \mathrm{P}_{1}\right)$ and hypertension $\left(\mathrm{S}_{1} \mathrm{P}_{3}\right)$ in rat. PLoS One 7:e52985.

Gergely P, Nuesslein-Hildesheim B, Guerini D, Brinkmann V, Traebert M, Bruns C, Pan S, Gray NS, Hinterding K, Cooke NG, et al. (2012) The selective sphingosine 1-phosphate receptor modulator BAF312 redirects lymphocyte distribution and has species-specific effects on heart rate. Br J Pharmacol 167:1035-1047.

Hindryckx P, Novak G, Vande Casteele N, Khanna R, Laukens D, Jairath V, and Feagan BG (2017) Incidence, prevention and management of anti-drug antibodies against therapeutic antibodies in inflammatory bowel disease: a practical overview [published correction appears in Drugs 2017;77:379]. Drugs 77:363-377.

Karuppuchamy T, Behrens EH, González-Cabrera P, Sarkisyan G, Gima L, Boyer JD, Bamias G, Jedlicka P, Veny M, Clark D, et al. (2017) Sphingosine-1-phosphate receptor-1 $\left(\mathrm{S}_{1} \mathrm{P}_{1}\right)$ is expressed by lymphocytes, dendritic cells, and endothelium and modulated during inflammatory bowel disease. Mucosal Immunol 10:162-171.

Olivera P, Danese S, and Peyrin-Biroulet L (2017) Next generation of small molecules in inflammatory bowel disease. Gut 66:199-209.

Ostanin DV, Pavlick KP, Bharwani S, D’Souza D, Furr KL, Brown CM, and Grisham MB (2006) T cell-induced inflammation of the small and large intestine in immunodeficient mice. Am J Physiol Gastrointest Liver Physiol 290:G109-G119.

Park SW, Kim M, Brown KM, D'Agati VD, and Lee HT (2012) Inhibition of sphingosine 1-phosphate receptor 2 protects against renal ischemia-reperfusion injury. J Am Soc Nephrol 23:266-280.

Pavlick KP, Ostanin DV, Furr KL, Laroux FS, Brown CM, Gray L, Kevil CG, and Grisham MB (2006) Role of T-cell-associated lymphocyte function-associated antigen-1 in the pathogenesis of experimental colitis. Int Immunol 18:389-398.

Peyrin-Biroulet L, Christopher R, Behan D, and Lassen C (2017) Modulation of sphingosine-1-phosphate in inflammatory bowel disease. Autoimmun Rev 16:495-503.

Piali L, Froidevaux S, Hess P, Nayler O, Bolli MH, Schlosser E, Kohl C, Steiner B, and Clozel M (2011) The selective sphingosine 1-phosphate receptor 1 agonist ponesimod protects against lymphocyte-mediated tissue inflammation. J Pharmacol Exp Ther 337:547-556.

Sandborn WJ, Feagan BG, Wolf DC, D'Haens G, Vermeire S, Hanauer SB, Ghosh S Smith H, Cravets M, Frohna PA, et al.; TOUCHSTONE Study Group (2016) Ozanimod induction and maintenance treatment for ulcerative colitis. $N$ Engl $J$ Med 374:1754-1762.

Sanna MG, Liao J, Jo E, Alfonso C, Ahn MY, Peterson MS, Webb B, Lefebvre S, Chun J, Gray N, et al. (2004) Sphingosine 1-phosphate (S1P) receptor subtypes S1P1 and S1P3, respectively, regulate lymphocyte recirculation and heart rate. J Biol Chem 279:13839-13848.

Schwab SR and Cyster JG (2007) Finding a way out: lymphocyte egress from lymphoid organs. Nat Immunol 8:1295-1301.

Shiow LR, Rosen DB, Brdicková N, Xu Y, An J, Lanier LL, Cyster JG, and Matloubian M (2006) CD69 acts downstream of interferon-alpha/beta to inhibit S1P1 and lymphocyte egress from lymphoid organs. Nature 440:540-544.

Torres J, Mehandru S, Colombel JF, and Peyrin-Biroulet L (2017) Crohn's disease. Lancet 389:1741-1755.

Tran JQ, Hartung JP, Tompkins CA, and Frohna PA (2018) Effects of high- and low-fat meals on the pharmacokinetics of ozanimod, a novel sphingosine-1phosphate receptor modulator. Clin Pharmacol Drug Dev 7:634-640.

Ungaro R, Mehandru S, Allen PB, Peyrin-Biroulet L, and Colombel JF (2017) Ulcerative colitis. Lancet 389:1756-1770.

Zundler S and Neurath MF (2017) Novel insights into the mechanisms of gut homing and antiadhesion therapies in inflammatory bowel diseases. Inflamm Bowel Dis 23:617-627.

Address correspondence to: Hussien Al-Shamma, 6118 Nancy Ridge Drive, San Diego, CA 92121. E-mail: salshamma@beacon-discovery.com 